\title{
The intersection convolution of relations and the Hahn-Banach type theorems
}

\author{
by Árpád SzÁz (Debrecen)
}

\begin{abstract}
By introducing the intersection convolution of relations, we prove a natural generalization of an extension theorem of B. Rodríguez-Salinas and L. Bou on linear selections which is already a substantial generalization of the classical Hahn-Banach theorems. In particular, we give a simple neccesary and sufficient condition in terms of the intersection convolution of a homogeneous relation and its partial linear selections in order that every partial linear selection of this relation can have an extension to a total linear selection.
\end{abstract}

Introduction. The following dominated extension theorems were first proved by H. Hahn and S. Banach in 1927 and 1929, respectively, by making use of the ideas of F. Riesz and E. Helly. (See, for instance, Dieudonné $[4$, p. 136] and Fuchssteiner-Lusky [6, p. 72].)

THEOREM 1. If $p$ is a norm on a real vector space $X$ and $\varphi$ is a linear functional on a subspace $Z$ of $X$ such that $|\varphi(z)| \leq p(z)$ for all $z \in Z$, then $\varphi$ can be extended to a linear functional $f$ on $X$ such that $|f(x)| \leq p(x)$ for all $x \in X$.

THEOREM 2. If $p$ is a sublinear functional on a real vector space $X$ and $\varphi$ is a linear functional on a subspace $Z$ of $X$ such that $\varphi(z) \leq p(z)$ for all $z \in Z$, then $\varphi$ can be extended to a linear functional $f$ on $X$ such that $f(x) \leq p(x)$ for all $x \in X$.

Theorems 1 and 2 were later generalized to linear operators taking their values in normed spaces with the binary intersection property and ordered

1991 Mathematics Subject Classification: Primary 46A22; Secondary 26E25, 54C60, $54 \mathrm{C} 65$.

Key words and phrases: intersection convolution, additive and homogeneous relations, linear selections, binary intersection property, Hahn-Banach theorems.

This paper forms a part of the author's investigations on a new foundation of linear analysis [27] supported in part by the Hungarian National Science Foundation Grant OTKA T 016846. 
vector spaces with the least upper bound property, respectively. (See Nachbin [19] and Buskes [3, p. 26], for instance.)

However, these generalizations turned out to be particular cases of the following fundamental selection type extension theorem of B. RodríguezSalinas and L. Bou [22] whose partial converse was later proved by A. D. Ioffe [15].

THEOREM 3. If $F$ is sublinear relation from one vector space $X$ into another $Y$ and $\mathcal{A}$ is a translation invariant family of subsets of $Y$ with the binary intersection property such that $F(x) \in \mathcal{A}$ for all $x \in X$, then each linear function $\varphi$ from a subspace $Z$ of $X$ into $Y$ such that $\varphi(z) \in F(z)$ for all $z \in Z$ can be extended to a linear function $f$ from $X$ into $Y$ such that $f(x) \in F(x)$ for all $x \in X$.

Theorem 2 can be easily derived from Theorem 3 by using the fact that the relation $F$ defined by

$$
F(x)=[-p(-x), p(x)]
$$

for all $x \in X$ and the family $\mathcal{A}$ of all closed subintervals of the real line $\mathbb{R}$ have the required properties.

In the present paper, by introducing the intersection convolution $F * \varphi$ defined by

$$
(F * \varphi)(x)=\bigcap_{z \in Z}(F(x-z)+\varphi(z))
$$

for all $x \in X$, we shall easily prove the following natural generalization of Theorem 3.

THEOREM 4. If $F$ is a homogeneous relation from one vector space $X$ into another $Y$, then the following assertions are equivalent:

(1) if $\varphi$ is a linear function from a subspace $Z$ of $X$ into $Y$ such that $\varphi(z) \in F(z)$ for all $z \in Z$, then $(F * \varphi)(x) \neq \emptyset$ for all $x \in X \backslash Z$;

(2) if $\Phi$ is a linear relation from a subspace $Z$ of $X$ into $Y$ such that $\Phi(z) \subset F(z)$ for all $z \in Z$, then $\Phi$ can be extended to a linear relation $\Psi$ from $X$ into $Y$ such that $\Psi(x) \subset F(x)+\Phi(0)$ for all $x \in X$.

Theorem 3 can be easily derived from Theorem 4 by using the fact that any two members of the family $\{F(x-z)+\varphi(z)\}_{z \in Z}$ have a nonvoid intersection whenever the relation $F$ is in particular sublinear. The necessary prerequisites about relations will be briefly laid out in the next preparatory section.

1. A few basic facts about relations. A subset $F$ of a product set $X \times Y$ is usually called a relation or a multifunction between $X$ and $Y$. In particular, $F$ is called a function if $(x, y) \in F$ and $(x, z) \in F$ imply $y=z$. 
To make an easily visible distinction between relations and functions, the latter will usually be denoted by lower case letters.

If $F$ is a relation between $X$ and $Y$ and $x \in X$, then the set

$$
F(x)=\{y \in X:(x, y) \in F\}
$$

is called the relation class or the image of $x$ under $F$. If in particular $F$ is a function and $y \in F(x)$, then by identifying singletons with their elements we may simply write $F(x)=y$ in place of $F(x)=\{y\}$.

If $F$ is a relation between $X$ and $Y$, then the set

$$
D_{F}=\{x \in X: F(x) \neq \emptyset\}
$$

is called the domain of $F$. And if in particular $X=D_{F}$, then by adopting the functional point of view we say that $F$ is a relation from $X$ into $Y$.

If $F$ and $\Phi$ are relations from $X$ into $Y$ such that $\Phi \subset F$, or equivalently $\Phi(x) \subset F(x)$ for all $x \in X$, then we say that $\Phi$ is a selection relation or a multiselection of $F$. In contrast to these total selections, the selections of the restriction $F \mid Z=F \cap(Z \times Y)$ of $F$ to a subset $Z$ of $X$ will be called partial selections of $F$.

If $X$ is an additive group and $A, B \subset X$, then we set $A+B=\{x+y$ : $x \in A, y \in B\},-A=\{-x: x \in A\}$, and $A-B=A+(-B)$. Moreover, if in particular $X$ is a vector space over a field $K$, then we also write $\lambda A=$ $\{\lambda x: x \in A\}$ for all $\lambda \in K$.

A relation $F$ between two groups $X$ and $Y$ will be called superadditive (resp. subadditive) if

$$
F(x)+F(y) \subset F(x+y) \quad(\text { resp. } F(x+y) \subset F(x)+F(y))
$$

for all $x, y \in X$, and additive if it is both superadditive and subadditive.

Moreover, $F$ will be called odd if

$$
F(-x)=-F(x)
$$

for all $x \in X$. Note that $F$ is odd if and only if $-F(x) \subset F(-x)$ (or equivalently $F(-x) \subset-F(x))$ for all $x \in X$.

It can be easily shown that an odd superadditive relation between groups is necessarily additive. Moreover, the importance of odd superadditive relations is also apparent from the fact that a relation between groups $X$ and $Y$ is odd and superadditive if and only if it is a subgroup of the product group $X \times Y$. Therefore, the oddness is frequently included in the definition of additive relations. (See, for instance, MacLane [18, pp. 51 and 63].)

In particular, a relation $F$ between vector spaces $X$ and $Y$ over $K$ is called homogeneous if

$$
F(\lambda x)=\lambda F(x)
$$


for all $\lambda \in K \backslash\{0\}$ and $x \in X$. Note that $F$ is homogeneous if and only if $\lambda F(x) \subset F(\lambda x)$ (or equivalently $F(\lambda x) \subset \lambda F(x)$ ) for all $\lambda \in K \backslash\{0\}$ and $x \in X$.

Now, a homogeneous relation between vector spaces may be called superlinear, sublinear and linear if it is superadditive, subadditive and additive, respectively. Note that homogeneous relations are in particular odd. Therefore, superlinear relations are necessarily linear. Moreover, a relation $F$ between vector spaces $X$ and $Y$ is linear if and only if it is a subspace of the product space $X \times Y$. (See, for instance, Arens [2] and Száz-Száz [29].)

2. The intersection convolution and its inclusion properties. Motivated by the ordinary convolution of integrable functions and the infimal convolution of extended real-valued functions [25], we make the following

Definition 2.1. If $F$ is a relation from one group $X$ into another $Y$ and $\Phi$ is a relation from a subgroup $Z$ of $X$ into $Y$, then the relation $F * \Phi$ defined by

$$
(F * \Phi)(x)=\bigcap_{z \in Z}(F(x-z)+\Phi(z))
$$

for all $x \in X$ will be called the intersection convolution of $F$ and $\Phi$.

REMARK 2.2. The intersection convolution can be naturally extended to arbitrary relations between semigroups. It surely deserves a further investigation, together with the union convolution.

However, in the sequel we shall only need the particular case of Definition 2.1 when $\Phi$ is a selection relation of $F \mid Z$. Namely, we shall only be interested in the extension of $\Phi$ to a suitable selection relation of $F+\Phi(0)$.

THEOREM 2.3. If $F$ is a relation from one group $X$ into another $Y$ and $\Phi$ is a relation from a subgroup $Z$ of $X$ into $Y$, then

$$
F * \Phi \subset F+\Phi(0) \text { and } F * \Phi \mid Z \subset F(0)+\Phi .
$$

Proof. By Definition 2.1,

$$
(F * \Phi)(x) \subset F(x-0)+\Phi(0)=(F+\Phi(0))(x)
$$

for all $x \in X$ and

$$
(F * \Phi)(z) \subset F(z-z)+\Phi(z)=(F(0)+\Phi)(z)
$$

for all $z \in Z$.

An immediate consequence of Theorem 2.3 is

Corollary 2.4. If $F$ and $\Phi$ are as in Theorem 2.3, then $F * \Phi \subset F$ if $\Phi(0)=\{0\}$ and $F * \Phi \mid Z \subset \Phi$ if $F(0)=\{0\}$.

We can also easily prove 
THEOREM 2.5. If $F$ is a relation from one group $X$ into another $Y$, and moreover $Z$ is a subgroup of $X$ and $\Phi$ is a subadditive selection relation of $F \mid Z$, then

$$
\Phi \subset F * \Phi \mid Z .
$$

Moreover, if $F(0)=\{0\}$, then this inclusion turns into equality.

Proof. If $z \in Z$, then

$$
\Phi(z)=\Phi(z-w+w) \subset \Phi(z-w)+\Phi(w) \subset F(z-w)+\Phi(w)
$$

for all $w \in Z$, that is, $\Phi \subset F * \Phi \mid Z$. If $F(0)=\{0\}$, then by Corollary 2.4 the converse inclusion is also true.

An immediate consequence of Theorem 2.5 is

Corollary 2.6. If $F$ is a relation from one group $X$ into another $Y$ such that $F(0)=\{0\}$ and $\Phi$ is a subadditive selection relation of $F$, then $\Phi=F * \Phi$.

In particular, we also have

Corollary 2.7. If $F$ is a subadditive relation from one group $X$ into another $Y$ such that $F(0)=\{0\}$, then $F=F * F$.

We can also easily prove

THEOREM 2.8. If $F$ is a relation from one group $X$ into another $Y$, and moreover $Z$ is a subgroup of $X$ and $\Phi$ is an additive selection relation of $F \mid Z$, then $\Phi$ is also a selection relation of $F+\Phi(0) \mid Z$ and

$$
F * \Phi=(F+\Phi(0)) * \Phi .
$$

Proof. In this case

$$
\Phi(z)=\Phi(z)+\Phi(0) \subset F(z)+\Phi(0)=(F+\Phi(0))(z)
$$

for all $z \in Z$ and

$$
\begin{aligned}
(F * \Phi)(x) & =\bigcap_{z \in Z}(F(x-z)+\Phi(z))=\bigcap_{z \in Z}(F(x-z)+\Phi(0)+\Phi(z)) \\
& =\bigcap_{z \in Z}((F+\Phi(0))(x-z)+\Phi(z))=((F+\Phi(0)) * \Phi)(x)
\end{aligned}
$$

for all $x \in X$.

3. Further inclusion properties of the intersection convolution. From Theorem 2.5, by using Definition 2.1, we easily get

THEOREM 3.1. If $F$ is a relation from one group $X$ into another $Y$, and $Z$ is a subgroup of $X$, and $\Phi$ is a subadditive selection relation of $F \mid Z$ and $\Psi$ is a subadditive selection relation of $F$ such that $\Phi=\Psi \mid Z$, then

$$
\Psi \subset F * \Phi \text {. }
$$


P r o of. In this case,

$$
\begin{aligned}
\Psi(x) & \subset(F * \Psi)(x)=\bigcap_{z \in X}(F(x-z)+\Psi(z)) \\
& \subset \bigcap_{z \in Z}(F(x-z)+\Psi(z))=\bigcap_{z \in Z}(F(x-z)+\Phi(z))=(F * \Phi)(x)
\end{aligned}
$$

for all $x \in X$.

An immediate consequence of Theorem 3.1 is

COROLlary 3.2. If $F$ and $\Phi$ are as in Theorem 3.1 and $\Phi$ can be extended to a subadditive selection relation of $F$, then $(F * \Phi)(x) \neq \emptyset$ for all $x \in X$.

Hence, by using Theorem 2.8, we also get

COROllary 3.3. If $F$ is a relation from one group $X$ into another $Y$, and moreover $Z$ is a subgroup of $X$ and $\Phi$ is an additive selection relation of $F \mid Z$ such that $\Phi$ can be extended to a subadditive selection relation of $F+\Phi(0)$, then $(F * \Phi)(x) \neq \emptyset$ for all $x \in X$.

Proof. In this case $\Phi$ is also a selection relation of $F+\Phi(0) \mid Z$ and

$$
(F * \Phi)(x)=((F+\Phi(0)) * \Phi)(x) \neq \emptyset
$$

for all $x \in X$ by Theorem 2.8 and Corollary 3.2.

An immediate consequence of Theorem 3.1 and Corollary 2.4 is

COROLlary 3.4. If $F$ is a subadditive relation from one group $X$ into another $Y$ such that $F(0)=\{0\}$ and $Z$ is a subgroup of $X$, then $F=$ $F *(F \mid Z)$.

Complementing Theorems 2.3 and 2.8, we can also prove

THEOREM 3.5. If $F$ is a superadditive relation from one group $X$ into another $Y$, and moreover $Z$ is a subgroup of $X$ and $\Phi$ is a selection relation of $F \mid Z$ such that $0 \in \Phi(0)$, then

$$
F=F+\Phi(0) .
$$

Proof. In this case,

$$
F(x) \subset F(x)+\Phi(0)=(F+\Phi(0))(x)
$$

and

$$
(F+\Phi(0))(x)=F(x)+\Phi(0) \subset F(x)+F(0) \subset F(x)
$$

for all $x \in X$.

THEOREM 3.6. If $F$ is a superadditive relation from one group $X$ into another $Y$, and moreover $Z$ is a subgroup of $X$ and $\Phi$ is a subadditive selection relation of $F \mid Z$, then

$$
F * \Phi=F+\Phi(0) \text {. }
$$


Proof. If $x \in X$, then

$$
\begin{aligned}
(F+\Phi(0))(x) & =F(x)+\Phi(0) \subset F(x)+\Phi(-z)+\Phi(z) \\
& \subset F(x)+F(-z)+\Phi(z) \subset F(x-z)+\Phi(z)
\end{aligned}
$$

for all $z \in Z$, that is, $(F+\Phi(0))(x) \subset(F * \Phi)(x)$. By Theorem 2.3, the converse inclusion is always true.

An immediate consequence of Theorems 3.5 and 3.6 is

COROLlary 3.7. If $F$ and $\Phi$ are as in Theorem 3.6 and $0 \in \Phi(0)$, then $F=F * \Phi$.

In particular, we also have

COROLlaRY 3.8. If $F$ is an additive relation from one group $X$ into another $Y$ such that $0 \in F(0)$ and $Z$ is a subgroup of $X$, then $F=F *(F \mid Z)$.

Corollary 3.9. If $F$ is an additive relation from one group $X$ into another $Y$ such that $0 \in F(0)$, then $F=F * F$.

4. Additivity and homogeneity properties of the intersection convolution. Since the ordinary convolution of integrable functions inherits several useful regularity properties of its factors, it may be expected that the relation $F * \Phi$ also inherits some algebraic properties of $F$ and $\Phi$.

THEOREM 4.1. If $F$ is a relation from one group $X$ into another $Y$ and $\Phi$ is an odd additive relation from a subgroup $Z$ of $X$ into $Y$, then

$$
(F * \Phi)(x+z)=(F * \Phi)(x)+\Phi(z)
$$

for all $x \in X$ and $z \in Z$.

Proof. Set $G=F * \Phi$. If $x \in X$ and $z \in Z$, then $G(x)+\Phi(z) \subset F(x-(w-z))+\Phi(w-z)+\Phi(z)=F((x+z)-w)+\Phi(w)$ for all $w \in Z$. Therefore

$$
G(x)+\Phi(z) \subset G(x+z) .
$$

Hence, $G(x+z)+\Phi(-z) \subset G(x)$, and thus the converse inclusion is also true:

$$
G(x+z) \subset G(x)-\Phi(-z)=G(x)+\Phi(z) .
$$

From Theorems 4.1 and 2.5, in particular we also have

COROLlary 4.2. If $F$ is a relation from one group $X$ into another $Y$ such that $F(0)=\{0\}$, and moreover $Z$ is a subgroup of $X$ and $\varphi$ is an additive selection function of $F \mid Z$, then

$$
(F * \varphi)(x+z)=(F * \varphi)(x)+(F * \varphi)(z)
$$

for all $x \in X$ and $z \in Z$. 
Whenever the groups $X$ and $Y$ are commutative, we also have

THEOREM 4.3. If $F$ is an odd relation from one Abelian group $X$ into another $Y$ and $\Phi$ is an odd relation from a subgroup $Z$ of $X$ into $Y$, then the relation $F * \Phi$ is also odd.

The proof is obvious from the $\lambda=-1$ case of the proof of

THEOREM 4.4. If $F$ is a homogeneous relation from one vector space $X$ into another $Y$ and $\Phi$ is a homogeneous relation from a subspace $Z$ of $X$ into $Y$, then the relation $F * \Phi$ is also homogeneous.

Proof. Set $G=F * \Phi$. If $\lambda \in K \backslash\{0\}$ and $x \in X$, then

$$
\begin{aligned}
\lambda G(x) & \subset \lambda\left(F\left(x-\lambda^{-1} z\right)+\Phi\left(\lambda^{-1} z\right)\right) \\
& =\lambda F\left(x-\lambda^{-1} z\right)+\lambda \Phi\left(\lambda^{-1} z\right)=F(\lambda x-z)+\Phi(z)
\end{aligned}
$$

for all $z \in Z$. Therefore, $\lambda G(x) \subset G(\lambda x)$, and thus $G$ is homogeneous.

EXAMPLE 4.5. Note that if

$$
\varphi=\{(x, x): x \in \mathbb{R}\} \text { and } \Phi=\{0\} \times \mathbb{R},
$$

then $F=\varphi \cup \Phi$ is a homogeneous relation on $\mathbb{R}$ such that $\varphi$ and $\Phi$ are the only partial linear selection relations of $F$. Moreover,

$$
F * \varphi=\varphi \text { and } F * \Phi=\mathbb{R}^{2} .
$$

But the partial linear selection relation $\Phi$ can only be extended to a total linear selection relation of $F+\Phi(0)=\mathbb{R}^{2}$.

Therefore, in the forthcoming extension theorems we shall rather give some necessary and sufficient conditions in order that a partial linear selection relation $\Phi_{0}$ of a homogeneous relation $F$ can be extended to a total linear selection relation $\Phi$ of $F+\Phi_{0}(0)$. Note that if in particular $\Phi_{0}$ is a function, then so is $\Phi$.

5. Linear selections of homogeneous relations. By using the results of the previous sections, we can easily prove the next simple extension theorem.

THEOREM 5.1. If $F$ is a homogeneous relation from one vector space $X$ into another $Y$, and moreover $Z$ is a subspace of $X$ such that $\operatorname{codim}(Z)=1$ and $\Phi_{0}$ is a linear selection relation of $F \mid Z$, then the following assertions are equivalent:

(1) $\left(F * \Phi_{0}\right)(x) \neq \emptyset$ for some $x \in X \backslash Z$;

(2) there exists a linear selection relation $\Phi$ of $F+\Phi_{0}(0)$ such that $\Phi_{0}=$ $\Phi \mid Z$. 
Proof. Set $G=F * \Phi_{0}$. If (1) holds, then there exist $x_{0} \in X \backslash Z$ and $y_{0} \in Y$ such that $y_{0} \in G\left(x_{0}\right)$. We have $X=K x_{0} \oplus Z$ because $\operatorname{codim}(Z)=1$.

Define a relation $\Phi$ from $X$ into $Y$ by

$$
\Phi\left(\lambda x_{0}+z\right)=\lambda y_{0}+\Phi_{0}(z)
$$

for all $\lambda \in K$ and $z \in Z$. The definition is clearly correct. Moreover, $\Phi$ is a linear relation from $X$ into $Y$ such that $\Phi_{0}=\Phi \mid Z$.

On the other hand, by Theorems 4.4, 4.1 and 2.3,

$$
\begin{aligned}
\Phi\left(\lambda x_{0}+z\right) & =\lambda y_{0}+\Phi_{0}(z) \subset \lambda G\left(x_{0}\right)+\Phi_{0}(z)=G\left(\lambda x_{0}\right)+\Phi_{0}(z) \\
& =G\left(\lambda x_{0}+z\right) \subset F\left(\lambda x_{0}+z\right)+\Phi_{0}(0)
\end{aligned}
$$

for all $\lambda \in K \backslash\{0\}$ and $z \in Z$. Since by Theorems 2.5 and 2.3 we have

$$
\Phi(z)=\Phi_{0}(z) \subset G(z) \subset F(z)+\Phi_{0}(0)
$$

for all $z \in Z$, it is clear that $\Phi$ is a selection relation of $F+\Phi_{0}(0)$, that is, (2) holds.

Conversely, if (2) holds, then by Corollary 3.3 we have $\left(F * \Phi_{0}\right)(x) \neq \emptyset$ for all $x \in X$, showing (1).

From Theorem 5.1, by using the Hausdorff maximality principle, we now easily get the following more general extension theorem.

THEOREM 5.2. If $F$ is a homogeneous relation from one vector space $X$ into another $Y$, and moreover $Z_{0}$ is a subspace of $X$ and $\Phi_{0}$ is a linear selection relation of $F \mid Z_{0}$, then $(2) \Rightarrow(1)$, where:

(1) there exists a linear selection relation $\Phi$ of $F+\Phi_{0}(0)$ such that $\Phi_{0}=$ $\Phi \mid Z_{0}$;

(2) if $\Phi \subset F+\Phi_{0}(0)$ is a linear relation such that $\Phi_{0}=\Phi \mid Z_{0}$, then $(F * \Phi)(x) \neq \emptyset$ for all $x \in X \backslash D_{\Phi}$.

Pr o of. Denote by $\mathcal{F}$ the family of all linear relations $\Psi \subset F+\Phi_{0}(0)$ such that $\Phi_{0}=\Psi \mid Z_{0}$. It is a nonvoid set partially ordered by inclusion. Namely, by Theorem 2.8, we have $\Phi_{0} \in \mathcal{F}$. Therefore, by the Hausdoff maximality principle, there exists a maximal totally ordered subset $\mathcal{G}$ of $\mathcal{F}$.

Define $\Phi=\bigcup \mathcal{G}$. Since for any $\Psi_{1}, \Psi_{2} \in \mathcal{G}$ we have either $\Psi_{1} \subset \Psi_{2}$ or $\Psi_{2} \subset \Psi_{1}$, it is clear that $\Phi$ is a linear relation from a certain subspace $Z$ of $X$ into $Y$. Moreover, since for any $\Psi \in \mathcal{G}$ we have $\Phi_{0}=\Psi \mid Z_{0}$ and $\Psi \subset F+\Phi_{0}(0)$, it is clear that $\Phi_{0}=\Phi \mid Z_{0}$ and $\Phi \subset F+\Phi_{0}(0)$.

Therefore, we need only show that if (2) holds, then $Z=X$. Assume that, on the contrary, there exists an $x_{0} \in X$ such that $x_{0} \notin Z$. Then, by Theorem 2.8 and (2), we have

$$
\left(\left(F+\Phi_{0}(0)\right) * \Phi\right)\left(x_{0}\right)=((F+\Phi(0)) * \Phi)\left(x_{0}\right)=(F * \Phi)\left(x_{0}\right) \neq \emptyset .
$$


Therefore, by Theorem 5.1, there exists a linear selection relation $\Psi$ of the restriction of the homogeneous relation

$$
F+\Phi_{0}(0)=F+\Phi_{0}(0)+\Phi_{0}(0)=\left(F+\Phi_{0}(0)\right)+\Phi(0)
$$

to the subspace $\Omega=K x_{0} \oplus Z$ of $X$ such that $\Phi=\Psi \mid Z$, and hence $\Phi_{0}=$ $\Psi \mid Z_{0}$. But $\mathcal{G} \cup\{\Psi\}$ is a totally ordered subset of $\mathcal{F}$, and this contradicts the maximality of $\mathcal{G}$ since $\Psi \notin \mathcal{G}$.

An immediate consequence of Theorem 5.2 is

COROLlary 5.3. If $F$ is a homogeneous relation from one vector space $X$ into another $Y$ and $W$ is a subspace of $Y$ such that $W \subset F(0)$ and for every linear relation $\Phi \subset F+W$ with $\Phi(0)=W$ we have $(F * \Phi)(x) \neq \emptyset$ for all $x \in X \backslash D_{\Phi}$, then there exists a linear selection relation $\Phi$ of $F+W$ such that $\Phi(0)=W$.

Pr o of. In this case $\Phi_{0}=\{0\} \times W$ is a linear selection relation of $F \mid\{0\}$ such that $\Phi_{0}(0)=W$, and thus Theorem 5.2 can be applied.

6. Linear selections of linear relations. From Theorem 5.2, by using Theorem 3.5 and Corollary 3.7, we can easily get the following extension theorem.

THEOREM 6.1. If $F$ is a linear relation from one vector space $X$ into another $Y$, and moreover $Z$ is a subspace of $X$ and $\Phi_{0}$ is a linear selection relation of $F \mid Z$, then there exists a linear selection relation $\Phi$ of $F$ such that $\Phi_{0}=\Phi \mid Z$.

Proof. In this case, by Theorem 3.5,

$$
F=F+\Phi_{0}(0) \text {. }
$$

Therefore, if $\Phi \subset F+\Phi_{0}(0)$ is a linear relation, then by Corollary 3.7 we have

$$
(F * \Phi)(x)=F(x) \neq \emptyset
$$

for all $x \in X$. Thus, by Theorem 5.2 , there exists a linear selection relation $\Phi$ of $F=F+\Phi_{0}(0)$ such that $\Phi_{0}=\Phi \mid Z$.

REMARK 6.2. It is a curious fact that, in contrast to Theorem 5.2, the above theorem can also be proved directly by taking a Hamel basis of $Z$ and enlarging it to a Hamel basis of $X$.

From Theorem 6.1, we at once get

COROLlary 6.3. If $F$ is a linear relation from one vector space $X$ into another $Y$, and moreover $Z$ is a subspace of $X$ and $\varphi_{0}$ is a linear selection function of $F \mid Z$, then there exists a linear selection function $\varphi$ of $F$ such that $\varphi_{0}=\varphi \mid Z$. 
REMARK 6.4. Hence, in particular, each linear relation has a linear selection function.

From Corollary 6.3, we also easily get the following more delicate

COROLlary 6.5. If $F$ is a linear relation from one vector space $X$ into another $Y$, then there exists a linear selection function $\varphi$ of $F$ such that $\varphi \circ F^{-1}$ is a function.

Proof. Note that $F^{-1}(0)$ is a subspace of $X$. Moreover, $x \in F^{-1}(0)$ implies $0 \in F(x)$. Therefore, $\varphi_{0}=F^{-1}(0) \times\{0\}$ is a linear selection function of $F \mid F^{-1}(0)$. Thus, by Corollary 6.3, there exists a linear selection function $\varphi$ of $F$ such that $\varphi_{0}=\varphi \mid F^{-1}(0)$. Hence, $\left(\varphi \circ F^{-1}\right)(0)=\{0\}$, and thus the linear relation $\varphi \circ F^{-1}$ is also a function.

REMARK 6.6. For the above linear functions $\varphi$ and $f=\varphi \circ F^{-1}$, we can also prove that $F=f^{-1} \circ \varphi$.

From Theorem 5.2, we at once get the following particular extension theorem.

THEOREM 6.7. If $F$ is a homogeneous relation from one vector space $X$ into another $Y$, and moreover $Z$ is a subspace of $X$ and $\varphi_{0}$ is a linear selection function of $F \mid Z$, then $(2) \Rightarrow(1)$, where:

(1) there exists a linear selection relation $\varphi$ of $F$ such that $\varphi_{0}=\varphi \mid Z$;

(2) if $\varphi \subset F$ is a linear function such that $\varphi_{0}=\varphi \mid Z$, then $(F * \varphi)(x) \neq \emptyset$ for all $x \in X \backslash D_{\varphi}$.

Proof. To derive this from Theorem 5.2, note that now we have $F=$ $F+\varphi_{0}(0)$. Moreover, if $\Phi$ is a linear relation such that $\varphi_{0}=\Phi \mid Z$, then $\Phi$ is necessarily a function.

An immediate consequence of Theorem 6.7 is

COROLlary 6.8. If $F$ is a homogeneous relation from one vector space $X$ into another $Y$ such that $0 \in F(0)$ and for every linear function $\varphi \subset F$ we have $(F * \varphi)(x) \neq \emptyset$ for all $x \in X \backslash D_{\varphi}$, then there exists a linear selection function $\varphi$ of $F$.

Moreover, from Theorem 6.7, by using Remark 6.4 and Corollary 3.2, we get the following universal extension theorem.

THEOREM 6.9. If $F$ is a homogeneous relation from one vector space $X$ into another $Y$, then the following assertions are equivalent:

(1) if $\varphi \subset F$ is a linear function, then $(F * \varphi)(x) \neq \emptyset$ for all $x \in X \backslash D_{\varphi}$;

(2) if $\Phi_{0} \subset F$ is a linear relation, then there exists a linear selection relation $\Phi$ of $F+\Phi_{0}(0)$ such that $\Phi_{0}=\Phi \mid D_{\Phi_{0}}$. 
Proof. If $\Phi_{0}$ is a linear relation from a subspace $Z$ of $X$ into $Y$, then by Remark 6.4 there exists a linear selection function $\varphi_{0}$ of $\Phi_{0}$. Hence, if $\Phi_{0} \subset F$ and (1) holds, then by Theorem 6.7 there exists a linear selection function $\varphi$ of $F$ such that $\varphi_{0}=\varphi \mid Z$.

Now, $\Phi=\varphi+\Phi_{0}(0)$ is a linear relation from $X$ into $Y$ such that

$$
\Phi(x)=\varphi(x)+\Phi_{0}(0) \subset F(x)+\Phi_{0}(0)=\left(F+\Phi_{0}(0)\right)(x)
$$

for all $x \in X$ and

$$
\Phi(z)=\varphi(z)+\Phi_{0}(0)=\varphi_{0}(z)+\Phi_{0}(0)=\Phi_{0}(z)
$$

for all $z \in Z$. Therefore, (2) holds.

The converse implication $(2) \Rightarrow(1)$ is an immediate consequence of a particular case of Corollary 3.2. Namely, if (2) holds, then every linear function $\varphi \subset F$ can be extended to a linear selection function of $F$.

7. Linear selections of sublinear relations. To provide an applicable sufficient condition for (1) of Theorem 6.9 to hold, we shall need the binary intersection property of Nachbin [19] which is closely related to Riesz's characterization of compactness and Cantor's characterization of completeness [17, pp. 136 and 193].

However, in contrast to Nachbin's original terminology, but in accordance with the standard definition of the finite intersection property, we adhere to the following

Definition 7.1. A family $\mathcal{A}$ of sets will be said to have the binary intersection property if $A \cap B \neq \emptyset$ for all $A, B \in \mathcal{A}$.

The importance of the binary intersection property for extension of partial linear selection relations of sublinear relations is already apparent from

THEOREM 4.7. If $F$ is an odd subadditive relation from one Abelian group $X$ into another $Y$, and moreover $Z$ is a subgroup of $X$ and $\Phi$ is an odd subadditive selection relation of $F \mid Z$, then the family

$$
\{F(x-z)+\Phi(z)\}_{z \in Z}
$$

has the binary intersection property for all $x \in X$.

Proof. If $z, w \in Z$ and $x \in X$, then

$$
\begin{aligned}
0 & \in(F(w-z)-\Phi(w-z))=F((x-z)-(x-w))-\Phi(w-z) \\
& \subset(F(x-z)-F(x-w))-(\Phi(w)-\Phi(z)) \\
& =(F(x-z)+\Phi(z))-(F(x-w)+\Phi(w)) .
\end{aligned}
$$

Therefore, we also have

$$
(F(x-z)+\Phi(z)) \cap(F(x-w)+\Phi(w)) \neq \emptyset .
$$


In view of Nachbin's fundamental investigations concerning the binary intersection property, it seems appropriate to introduce the following

Definition 7.3.A family $\mathcal{A}$ of subsets of a set $X$ will be called a Nachbin system in $X$ if every subfamily of $\mathcal{A}$ having the binary intersection property has a nonvoid intersection.

REMARK 7.4. Quite similarly, a family of subsets of a set may be called a Riesz system if every subfamily having the finite intersection property has a nonvoid intersection.

Moreover, a family of subsets of a uniform space may be called a Cantor system if every subfamily containing small sets and having the finite intersection property has a nonvoid intersection.

This terminology, according to Kelley [17, pp. 136 and 193], allows us to briefly state that a topological (resp. uniform) space is compact (resp. complete) if and only if the family of its closed subsets forms a Riesz (resp. Cantor) system.

Now, by using Theorem 7.2, from Theorem 6.9 we can easily get the following generalization of a fundamental extension theorem of RodríguezSalinas and Bou $[22]$. (See also the implication $(a) \Rightarrow(b)$ in Theorem B of Ioffe [15].)

THEOREM 7.5. If $F$ is a sublinear relation from one vector space $X$ into another $Y$ and $\mathcal{A}$ is a translation invariant Nachbin system in $Y$ such that $F(x) \in \mathcal{A}$ for all $x \in X$, then for every linear subspace $Z$ of $X$ and every linear selection relation $\Phi_{0}$ of $F \mid Z$ there exists a linear selection relation $\Phi$ of $F+\Phi_{0}(0)$ such that $\Phi_{0}=\Phi \mid Z$.

Proof. If $\varphi \subset F$ is a linear function and $x \in X$, then by Theorem 7.2 the family $\{F(x-z)+\varphi(z)\}_{z \in D_{\varphi}}$ has the binary intersection property. Since this family is a subfamily of $\mathcal{A}$, it is clear that

$$
(F * \varphi)(x)=\bigcap_{z \in D_{\varphi}}(F(x-z)+\varphi(z)) \neq \emptyset .
$$

Therefore, by Theorem 6.9, the required assertion is true.

An immediate consequence of Theorem 7.5 is

Corollary 7.6. If $F$ and $\mathcal{A}$ are as in Theorem 7.5, then for every linear subspace $Z$ of $X$ and every linear selection function $\varphi_{0}$ of $F \mid Z$ there exists a linear selection function $\varphi$ of $F$ such that $\varphi_{0}=\varphi \mid Z$.

In particular, we also have

Corollary 7.7. If $F$ and $\mathcal{A}$ are as in Theorem 7.5 and $0 \in F(0)$, then there exists a linear selection function $\varphi$ of $F$. 
REMARK 7.8. To feel the range of the applicability of the above results the reader is referred to Nachbin [19], Rodríguez-Salinas and Bou [22] and Ioffe [15].

\section{References}

[1] J. Abreu and A. Etchebery, Hahn-Banach and Banach-Steinhaus theorems for convex processes, Period. Math. Hungar. 20 (1989), 289-297.

[2] R. Arens, Operational calculus of linear relations, Pacific J. Math. 11 (1961), 9-23.

[3] G. Buskes, The Hahn-Banach Theorem surveyed, Dissertationes Math. 327 (1993).

[4] J. Dieudonné, History of Functional Analysis, North-Holland Math. Stud. 49, North-Holland, Amsterdam, 1981.

[5] B. Fuchssteiner und J. Horváth, Die Bedeutung der Schnitteigenschaften beim Hahn-Banachschen Satz, Jahrbuch Überblicke Math. (BI, Mannheim) 1979, 107121.

[6] B. Fuchssteiner and W. Lusky, Convex Cones, North-Holland Math. Stud. 56, North-Holland, Amsterdam, 1981.

[7] Z. Gajda, A. Smajdor and W. Smajdor, A theorem of the Hahn-Banach type and its applications, Ann. Polon. Math. 57 (1992), 243-252.

[8] G. Godini, Set-valued Cauchy functional equation, Rev. Roumaine Math. Pures Appl. 20 (1975), 1113-1121.

[9] D. B. Goodner, Projections in normed linear spaces, Trans. Amer. Math. Soc. 69 (1950), 89-108.

[10] M. Hasumi, The extension property of complex Banach spaces, Tôhoku Math. J. 10 (1958), 135-142.

[11] L. Holá and P. Maličký, Continuous linear selectors of linear relations, Acta Math. Univ. Comenian. 48-49 (1986), 153-157.

[12] J. Horváth, Some selected results of professor Baltasar Rodríguez-Salinas, Rev. Mat. Univ. Complut. Madrid 9 (1996), 23-72.

[13] O. Hustad, A note on complex $\mathcal{P}_{1}$ spaces, Israel J. Math. 16 (1973), 117-119.

[14] A. W. Ingleton, The Hahn-Banach theorem for non-Archimedean-valued fields, Proc. Cambridge Philos. Soc. 48 (1952), 41-45.

[15] A. D. Ioffe, A new proof of the equivalence of the Hahn-Banach extension and the least upper bound properties, Proc. Amer. Math. Soc. 82 (1981), 385-389.

[16] J. L. Kelley, Banach spaces with the intersection property, Trans. Amer. Math. Soc. 72 (1952), 323-326.

[17] J. L. Kelley, General Topology, Van Nostrand Reinhold, New York, 1955

[18] S. Mac Lane, Homology, Springer, Berlin, 1963.

[19] L. Nachbin, A theorem of the Hahn-Banach type for linear transformations, Trans. Amer. Math. Soc. 68 (1950), 28-46.

[20] K. Nikodem, Additive selections of additive set-valued functions, Zb. Rad. Prirod.Mat. Fak. Univ. u Novom Sadu Ser. Mat. 18 (1988), 143-148.

[21] Zs. Páles, Linear selections for set-valued functions and extension of bilinear forms, Arch. Math. (Basel) 62 (1994), 427-432.

[22] B. Rodríguez-Salinas and L. Bou, A Hahn-Banach theorem for arbitrary vector spaces, Boll. Un. Mat. Ital. 10 (1974), 390-393.

[23] W. Smajdor, Subadditive and subquadratic set-valued functions, Prace Nauk. Uniw. Śląsk. Katowic. 889 (1987), 73 pp. 
[24] W. Smajdor and J. Szczawińska, A theorem of the Hahn-Banach type, Demonstratio Math. 28 (1995), 155-160.

[25] T. Strömberg, The operation of infimal convolution, Dissertationes Math. 352 (1996).

[26] Á. Száz, Pointwise limits of nets of multilinear maps, Acta Sci. Math. (Szeged) 55 (1991), 103-117.

[27] - Foundations of Linear Analysis, Inst. Mat. Inf., Lajos Kossuth University Debrecen 1996, 200 pp. (Unfinished lecture notes in Hungarian).

[28] Á. Száz and G. Száz, Additive relations, Publ. Math. Debrecen 20 (1973), 259-272.

[29] —, —, Linear relations, ibid. 27 (1980), 219-227.

[30] J. Zowe, Sandwich theorems for convex operators with values in an ordered vector space, J. Math. Anal. Appl. 66 (1978), 282-396.

Institute of Mathematics and Informatics

Lajos Kossuth University

H-4010 Debrecen, Pf. 12, Hungary

E-mail:szaz@math.klte.hu

Reçu par la Rédaction le 17.7.1997

Révisé le 12.3.1998 